Results 192 prescriptions corresponding to 112 patients were identified. 62 of $112(56 \%)$ were prescribed long-term azithromycin. Bronchiectasis (60\%), COPD (19\%), asthma (8\%), ILD (7\%), Bronchiolitis and others (6\%) were the variety of diseases for which Azithromycin was prescribed. 28\% had pseudomonas colonisation.

$51(46 \%)$ patients were prescribed long-term azithromycin. Of these, 21 (25\%) had been on azithromycin for less than 12 months.

Sixty eight $(82 \%)$ patients on long-term azithromycin had had LFTs and 3(3.5\%) had audiology testing in the preceding 12 months.21(25\%)had documented ECGs with Qtc interval.

No patients tested had hearing loss and no documented QTc prolongation.

The other common side effect noticed was GI upset in 6 patients (5.6\%).

The dosing was mostly $250 \mathrm{mg}$ three times a week $103(92 \%)$

There was also $500 \mathrm{mg}$ three times a week in $20(18 \%)$

H.influenzae (42\%)Staphylococcus aureus (21\%)Moraxella catarrhalis $(11 \%)$ coliform sp (10\%). We identified no new NTM in our Cohort.

Conclusion Although formal monitoring in this cohort was patchy, Significant documented adverse effects in this cohort were rare and optimal practice for long-term management of azithromycin use remains to be established.

\section{P112 DEEPER PHENOTYPING OF NON CF BRONCHIECTASIS THROUGH SPUTUM DIFFERENTIAL COUNTS}

${ }^{1} \mathrm{~J}$ Davison, ${ }^{1} \mathrm{G}$ Johnson, ${ }^{1} \mathrm{~K}$ Jiwa, ${ }^{1} \mathrm{~A}$ Cranson, ${ }^{1} \mathrm{R}$ Wake, ${ }^{1} \mathrm{~B}$ Hood, ${ }^{1} \mathrm{~T}$ Small, ${ }^{2} \mathrm{~J}$ Birch, ${ }^{2} \mathrm{~A}$ Fisher, ${ }^{2} \mathrm{~A}$ De Soyza; ${ }^{1}$ The Newcastle upon Tyne Hospitals NHS Foundation Trust, Newcastle upon Tyne, UK; ${ }^{2}$ Newcastle University, Newcastle upon Tyne, UK

\subsection{6/thoraxjnl-2013-204457.262}

Introduction and Objectives Non CF Bronchiectasis has diverse aetiologies. This includes idiopathic, systemic disease related and as a complication of asthma. Such diversity may be important in determining therapeutic strategies (personalised medicines) and may also be an important consideration in clinical trial design. This is increasingly relevant when neutrophil targeting or eosinophil targeted therapies are being developed. We hypothesised that patients could be phenotyped by sputum cytospins irrespective of suspected aetiology or disease severity.

Methods Patients underwent a standardised clinical phenotyping protocol including HRCT chest (Anwar et al 2013). Baseline therapy was recorded. Spontaneous sputa were collected in stable state and spirometry was undertaken according to guidelines. Sputum cell counts were calculated using standard methods with data expressed as medians and ranges.

Results Fifty three patients' data are reported. The M:F ratio was $1: 1.4$. The mean FEV1 predicted was $62 \%$, mean FEV1/VC ratio was $64 \%$. Forty three $(83 \%)$ were on inhaled corticosteroids and $24.5 \%$ had a historical diagnosis of asthma and /or ABPA. The predominant cell in sputa was neutrophils, median 94 (range 23-100\%), macrophages were the 2nd most prevalent cell type median 2.6 (range 0-75\%). Eosinophils showed a skewed distribution with median of 0.2 with a range of 0 $24.8 \%$. Four patients had sputum eosinophilia $>3 \%$. Of these, only 2 had a history of asthma and / or ABPA being diagnosed. Despite historical diagnoses of asthma and / or ABPA in 13 patients the eosinophil percentage was not statistically different to "non asthmatics". ( $P=0.59$ Chi Sq test $)$ This group included features of ABPA in 2 patients and significant atopy in another.
Conclusions Non CF bronchiectasis patients usually have sputum neutrophil dominance. Sputum eosinophilia is rare $(<10 \%$ of patients); however such patients may need alternative therapeutic strategies. Excluding bronchiectasis patients with a history of asthma from trials targeting neutrophils seems unnecessary. The neutrophilic predominant profiles in asthmatic bronchiectasis patients suggest either asthma misdiagnosis or that neutrophil predominant asthmatics may be more susceptible to developing bronchiectasis. Longitudinal studies are needed to determine if the sputum cell profiles are static in stable patients. These data may help develop a more personalised medicine approach in bronchiectasis.

\begin{tabular}{|c|c|c|c|c|}
\hline & NEUT & EOSIN & MACRO & LYMPH \\
\hline Median & 94.80 & 0.2 & 2.60 & 0.40 \\
\hline Max & 100.00 & 24.80 & 75.20 & 7.00 \\
\hline Min & 23.40 & 0.00 & 0.00 & 0.00 \\
\hline
\end{tabular}

\section{REFERENCES}

1. Anwar GA, et al. 2013 Phenotyping adults with non-cystic fibrosis bronchiectasis: A prospective observational cohort study. Respir Med. Jul;107(7):1001-7. doi: 10.1016/ j.rmed.2013.04.013. Epub 2013 May 11.

\section{P113 FREQUENT CO-DETECTION OF NON-TUBERCULOUS MYCOBACTERIA WITH OTHER MICROBES IN A UK CLINIC POPULATION: WHAT ARE THE IMPLICATIONS FOR TREATMENT?}

T Mahungu, M Yousefzadeh, N Shah, J Reading, J Hurst, S Burns, J Goldring, I Abubakar, I Cropley, S Hopkins, M Lipman; Royal Free London NHS Foundation Trust, London, United Kingdom

\subsection{6/thoraxjnl-2013-204457.263}

Introduction Non-Tuberculous Mycobacteria (NTM) are often isolated from patient samples, though their clinical relevance can be unclear. Treatment is not always effective and management decisions are usually based on repeat isolates with compatible clinical features. The presence of other micro-organisms, as well as the specific NTM itself, may be important. Here we report NTM and other microbe isolation frequency and their relationship to management decisions.

Methods All NTM samples isolated from liquid culture systems between $09 / 05 / 11$ and $03 / 04 / 13$ at our centre were identified using hospital pathology databases. Subject's negative mycobacterial cultures plus all positive relevant bacteria and virological isolates, as well as clinical history and progress were reviewed.

Results NTM were isolated on 257 occasions from 102 patients, who provided a total of 693 samples for mycobacterial culture. Adjusting for positive samples obtained within a month of each other, there were 170 isolates - 150 of which came from 90 patients' pulmonary samples. Common associated clinical conditions were non cystic fibrosis bronchiectasis $(28,31.1 \%)$, COPD (11, 12.2\%), and HIV infection $(6,6.7 \%)$. The most frequent lung isolate was Mycobacterium avium intracellulare Complex, MAC, (47.8\%), followed by M. fortuitum(14.4\%), M. gordonae (10\%), and M. kansasii (8.9\%). Seven (7.8\%) patients had multiple NTM species identified. $40(44.4 \%)$ of the pulmonary patients also had bacteria or fungi isolated from lung samples. Pseudomonas sp. were present in 12 (13.3\%), Haemophilus influenzae in $10(11.1 \%)$, and Staphylococcus aureus in 6 (6.7\%) 


\section{Correction: P112 Deeper phenotyping of non CF bronchiectasis through sputum differential counts}

Davison J, Johnson G, Jiwa K, et al. P112 Deeper phenotyping of non CF bronchiectasis through sputum differential counts. Thorax 2013;68:A125. doi: 10.1136/thoraxjnl-2013-204457.262.

The fourth author's name is incorrect. Instead of A Cranson, the correct spelling should be $A$ Cranston.

(c) Author(s) (or their employer(s)) 2018. No commercial re-use. See rights and permissions. Published by BMJ.

Thorax 2018;73:979. doi:10.1136/thoraxjnl-2013-204457.262corr1

D) Check for updates 